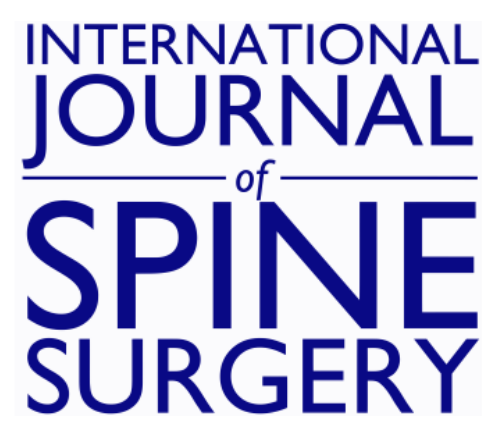

\title{
Spine Image Guidance and Robotics: Exposure, Education, Training, and the Learning Curve
}

Brendan F. Judy, Zachary Pennington, David Botros, Yohannes Tsehay, Srujan Kopparapu, Ann Liu, Nicholas Theodore and Hesham M. Zakaria

Int J Spine Surg 2021, 15 (s2) S28-S37

doi: https://doi.org/10.14444/8138

http://ijssurgery.com/content/15/s2/S28

This information is current as of April 26, 2023.

Email Alerts Receive free email-alerts when new articles cite this article. Sign up at: http://ijssurgery.com/alerts 


\title{
Spine Image Guidance and Robotics: Exposure, Education, Training, and the Learning Curve
}

\author{
BRENDAN F. JUDY, MD, ZACHARY PENNINGTON, BS, DAVID BOTROS, BS, YOHANNES TSEHAY, BS, \\ SRUJAN KOPPARAPU, BS, ANN LIU, MD, NICHOLAS THEODORE, MD, HESHAM M. ZAKARIA, MD \\ Department of Neurosurgery, Johns Hopkins Hospital, Baltimore, Maryland
}

\begin{abstract}
The use of intraoperative robotics and imaging for spine surgery has been shown to be safe, efficacious, and beneficial to patients, offering accurate placement of instrumentation, decreased operative time and blood loss, and improved postoperative outcomes. Despite these proven benefits, it has yet to be uniformly adopted. One of the major barriers for universal adoption of intraoperative robotics is the learning curve for this complex technology, in conjunction with a lack of formalized training. These same obstacles for universal adoption were faced in the introduction of surgical technology in other disciplines, and the use of this technology has become the standard of care in some of those specialties. Part of the success and widespread implementation of prior novel technology was the introduction of formalized training systems, which are currently lacking in advanced spine surgical technology. Therefore, the future success of intraoperative robotics and imaging for spine surgery depends on the creation of a formalized training system. We detail the best techniques for surgical pedagogy, as well as propose a comprehensive curriculum.
\end{abstract}

Special Issue Article

Keywords: navigation, robotic surgery, spine surgery

\section{INTRODUCTION}

Technological advances have always been the driving force for improving the care delivered during spine surgery, with overt examples including computed tomography (CT), magnetic resonance imaging, and fluoride positron emission tomography-CT. ${ }^{1}$ After the introduction of image-guided surgery of the spine in the late 1990s, the next phase in technological advancement for spine surgery was the implementation of intraoperative robotics coupled with real-time image guidance. Numerous commercial platforms are currently available, ${ }^{1,2}$ with proposed advantages including simplification of ergonomically challenging approaches, operator indefatigability, tremor elimination, increased reliability, and facilitation of minimally invasive techniques. ${ }^{2}$ Indeed, this technology has consistently been proven to be safe and efficacious in numerous meta-analyses, ${ }^{3}$ with specific outcomes including improved accuracy of pedicle screws, fewer proximal facet violations, decreased radiation exposure for surgeons and staff, decreased intraoperative blood loss, shorter postoperative stay, and lower revision rates. ${ }^{4-7}$ However, even given the mounting available evidence that this technology is safe and valuable to surgeons, it has not been widely adopted or become the standard of care. ${ }^{8-11}$

An unmodifiable barrier to universal adoption may be that the medical sciences have relatively longer technology lifecycles, especially compared with computing or automotive vehicle technology. ${ }^{12}$ In the technology adoption lifecycle, a model that describes the acceptance of a new product, image guidance and robotics are currently in the "chasm," which describes the gap between the early adopters and the market majority. ${ }^{13}$ Surgeons in general may be late adopters due to an understandable trepidation about a complex and unfamiliar technology that they may deem unnecessary; they know what works for them and are resistant to change, especially when considering pragmatic risk-reward comparisons and nostalgic biases. ${ }^{11}$ In 2001, Scott ${ }^{14}$ proposed a parabolic phenomenon for technologic adoption in medicine, outlining the progression from a promising idea to ubiquity (Figure 1). On this continuum, image guidance and robotics for spine surgery certainly have no dearth of encouraging reports. However, widespread enthusiasm is clearly lacking. Summarily, although this technolo- 


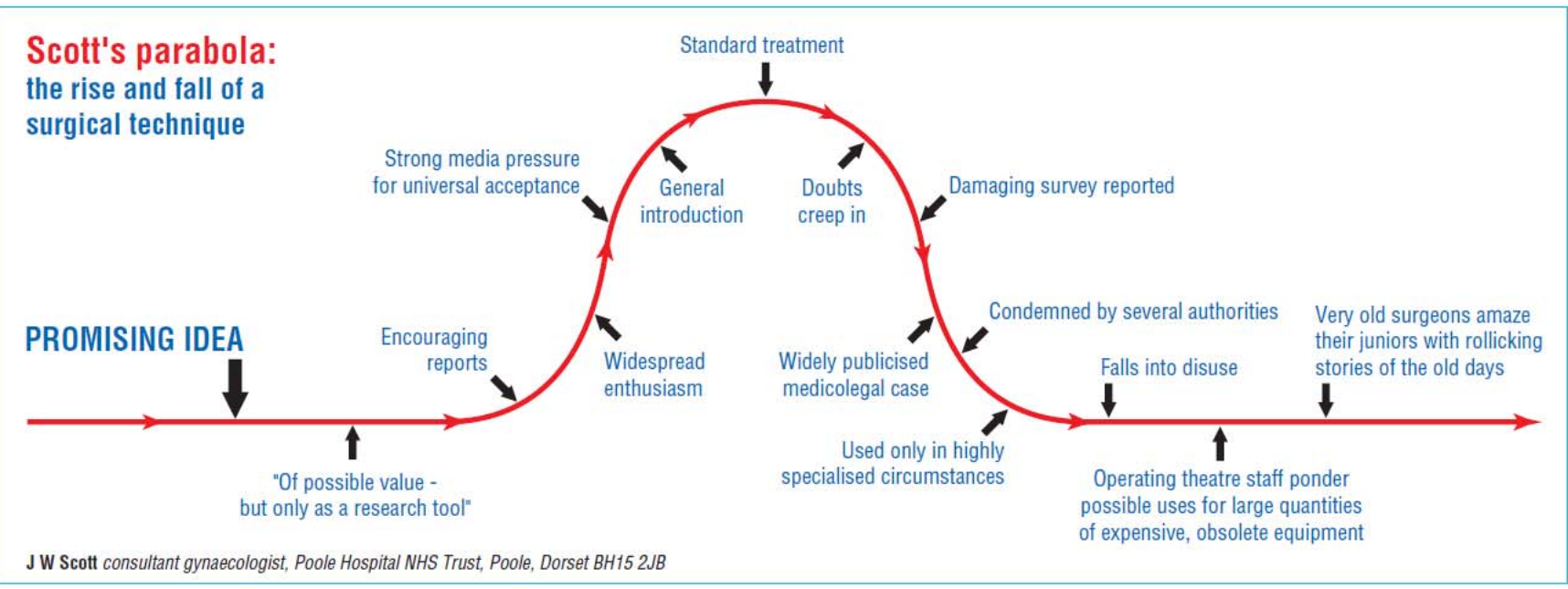

Figure 1. Scott's parabola.

gy has proven efficacy, it clearly has a long way to go before it becomes ubiquitous.

There are perhaps modifiable obstacles that prevent the transition of this beneficial technology to cross the chasm and move toward widespread adoption. Consistent evidence suggests that surgeons are loathe to use intraoperative robotics and image guidance during spine surgery because of a deficiency of training and exposure. This fact essentially establishes that the learning curve, inherent in all advanced technology, is a major barrier for adoption. ${ }^{11,15}$ In a global survey, Härtl et $\mathrm{al}^{16}$ noted that surgeons themselves cite inadequate training as a primary driver of nonusage of image guidance and found that a mere one-third of surgeons had ever participated in a computerassisted surgery training course. The lack of training leads to a lack of familiarity and a concern for disruption of operating room workflow, which hinders its acceptance even further. ${ }^{15,16}$ In teaching programs, there is the additional concern that trainees may foster reliance on this technology and downplay its potential to enhance the training experience. ${ }^{11}$ These attitudes stand in stark contrast to intraoperative cranial navigation, which has achieved universal adoption when available and has become an important tool for neurosurgical education and the standard of care for cranial surgery. ${ }^{17}$

Given the evidence that lack of adoption is directly related to training and education, comprehensive and thoughtful training is a prerequisite for universal adoption of this safe and expedient technology. Indeed, the success of this technology seems to be most closely tied to teaching how to use it. With education comes familiarity, an understanding of its advantages and disadvantages, as well as how to best incorporate it into a streamlined practice. In this manuscript, we extract lessons from other specialties during the incorporation of new technologies, summarize the evidence for the best way to teach a new technology or skill, and provide focused and practical advice on how to train in intraoperative spinal robotics and navigation.

\section{THE LAPAROSCOPIC JOURNEY AND BEYOND}

The technological journey of laparoscopic surgery is pertinent. ${ }^{14}$ In 1901 , the term laparoscopy was coined to describe the act of placing an endoscope within the peritoneal cavity, and the 1920s produced advancements that simplified its use, including the angled lens for lateral vision and an apparatus for peritoneal insufflation. ${ }^{18}$ The first laparoscopic cholecystectomy was performed in 1985, marking the first major surgical operation done laparoscopically and sparking a new era in general surgery. ${ }^{19}$ Although there was clear promise of its utility, a chief barrier for the widespread incorporation of the laparoscope was the steep learning curve, which is similar to the learning curve observed in intraoperative robotics and image guidance for spine surgery. General surgeons were unfamiliar with 2-dimensional video rendering, limited degrees of freedom of the instruments, lack of tactile feedback, and unstable camera platforms. ${ }^{19,20}$ To overcome this obstacle, the general surgery residency curriculum was modified to incorporate laparoscopy early in training; the 
Accreditation Council for Graduate Medical Education mandated standardized laparoscopic simulation and skills laboratories for all surgical residency programs, and this enhanced training experience was directly responsible for addressing the learning curve and played a role in catapulting laparoscopic surgery to the peak on Scott's parabola. ${ }^{21,22}$ The mantra, "See one, do one, teach one," coined over a century ago by William Halsted of Johns Hopkins, was modified to incorporate simulations, wherein the "do one" was first extensively performed on training models. ${ }^{22}$ The introduction of simulations also allowed the development of objective measurements of skill, introducing thresholds for competency before proceeding to live patients, such as with the McGill Inanimate System for Training and Evaluation of Laparoscopic Skills (MISTELS). ${ }^{23}$ Additionally, a Fundamentals of Laparoscopic Surgery curriculum was created to set standards for training. Therefore, based on the history and evolution of laparoscopy, the success of intraoperative navigation and robotics in spine surgery is wholly dependent on the ability to train surgeons and to incorporate that training early in the curriculum.

In the late 1990s, robotic surgery began offering novel solutions to plain laparoscopy. Perhaps the best-known surgical robot is the da Vinci Surgical System, whose inception was a Department of Defense project named AESOP. ${ }^{24}$ Although it took several iterations to reach its current form, the da Vinci was created to directly address the chief limitations of laparoscopy; namely, 3-dimensional visualization, fine movement control, and maneuverability. For example, the laparoscopic radical prostatectomy was not popularized due to difficulty in performing the vesico-urethral anastomosis. ${ }^{25} \mathrm{~A}$ mere decade after the first reported laparoscopic radical prostatectomy, its robotic counterpart with the da Vinci has become the standard of care in well-resourced countries. ${ }^{25}$ Both the da Vinci system and intraoperative robotics and image guidance for spine surgery have proven efficacy, are expensive, and have a steep learning curve. So how did the da Vinci become standard of care? Its success was related to the success of the laparoscope, in that there was a concerted effort to create training systems for this novel technology, combined with a decreasing price tag.

Indeed, the current iteration of spine robotics and navigation may still be cost prohibitive for wide- spread incorporation and training. This situation is most like that of the MAKO Robot, which is a CTguided robotic-assisted surgical system used in orthopaedics for hip and knee arthroplasty. ${ }^{26,27}$ Both technologies are proven efficacious but have yet to be widely incorporated due to cost. The price tag of the MAKO Robot may also create a negative feedback loop, preventing training, and thus lack of familiarity and further hesitancy for adoption.

To address the issue of robotic training for arthroplasty directly, there have been efforts to create formalized training modules. Just as there are modules for Fundamentals of Laparoscopic Surgery, there are also now Fundamentals of Robotic Surgery modules. ${ }^{28}$ Subsequently, different types of online training curricula, onsite training programs, and procedure-training simulators were developed on the foundation of these fundamentals. ${ }^{29}$ Certain neurosurgery residencies have independently identified the need for training in spinal robotics and navigation and have created their own structured training and certification programs. These programs incorporate reading materials, online training, and virtual simulations in the initial stage, which is then followed by more hands-on experience in the operating room. ${ }^{30}$ Thus, the educational arena of robotic surgery will continue to evolve as technological advancements develop and will play a pivotal role in pushing novel surgical systems up on Scott's parabola toward becoming standard of care.

\section{PEDAGOGY IN HEALTH CARE: APPLICATIONS TO SPINE SURGERY}

Now that the value of training has been emphasized, it is important to discuss how best to train in these technologies. Medical education in general, as inspired by Abraham Flexner and William Halsted, strongly emphasizes iterative clinical practice. ${ }^{31}$ Currently, a large body of evidence suggests that simulations may be the most efficient way to train..$^{22,32,33}$ The use of simulated experiences avoids ethical concerns of treating patients as "practice" in a traditional apprenticeship model, ${ }^{34}$ increases patients' support of trainee involvement in care, ${ }^{35}$ and may even reduce downstream health care costs. ${ }^{32}$ A simple example of this is simulated medical school clinical encounters, such as standardized patients or objective structured clinical exams, which have become required medical curricula, as their use has demonstrated a dose-response relationship with learning 
outcomes. ${ }^{36}$ A second example is that of advanced cardiovascular life support training, which involves standardized high-fidelity simulations; these simulations were not created for their own sake but because they have demonstrated improvements in provider care and patient outcomes during emergency situations. ${ }^{33}$

The importance of simulations is easily applied to surgical training, which already relies on deliberate repetition. ${ }^{22}$ A simulation of surgical techniques allows for virtually unlimited practice opportunities in a low-stress, supportive environment, which is essential for robust skill acquisition and long-term maintenance. ${ }^{36}$ There are several examples, ranging from low-fidelity (eg, simple knot tying, suture boards) to extremely high-fidelity (eg, cadaveric dissections) simulators. However, the validity and reliability of available simulators varies widely by surgical specialty and simulator type. ${ }^{37}$ Ultimately, the success of simulations hinges on measurable objective outcomes, integration into the curriculum, and standardized settings. To that end, work has been done to create standardized curricula and assessment tools for robotic surgery, emphasizing objective assessment of teachable skills, such as eliminating unnecessary maneuvers and respect for nearby tissue. ${ }^{38}$

Perhaps the most successful surgical simulators are low cost but high yield; for example, laparoscopic simulators, such as MISTELS, use simple but translatable tasks, such as peg transfers and pattern cutting, to practice transferable skills of tissue manipulation and visuospatial awareness. ${ }^{23}$ Although MISTELS is a lower-fidelity simulator, its ability to meet the previously mentioned components of simulation (ie, controlled setting, timely feedback, measurable outcomes, ability to increase difficulty) underlies its consistent validation as a learning and assessment tool for future laparoscopic surgeons. $^{22,23}$ The widespread availability of MISTEL stems from its proven efficacy and relatively low cost and is a critical component to the success of laparoscopic surgery. In terms of robotic surgery, a similar low-cost but high-yield simulator is extant and was key to the success of the da Vinci Surgical System. ${ }^{31}$ The Mimic dV-Trainer (Mimic Technologies Inc) has enabled training surgeons to practice transferable skills of a da Vinci Surgical System on a validated, lower-cost simulator. ${ }^{39}$ It has been proven efficacious, as the use of the Mimic dV-Trainer has been associated with shorter operative times in hernia repair cases. ${ }^{40}$

In stark contrast, neurosurgical spine simulators have been slow to develop, which is hindering the widespread adoption of intraoperative robotics and navigation. The lack of simulators may in part be due to their hypothetical cost, as well as their unknown reproducibility and efficacy. ${ }^{41,42}$ However, it is clear that the apprenticeship model is inadequate preparation for residents on these new technologies, ${ }^{42}$ as even attending neurosurgeons may not be completely familiar with the new system, and resident surgeons may quickly reach an artificial plateau created by their mentors. Therefore, the future success of intraoperative robotics and navigation depends on the creation of new low-cost and high-fidelity simulators. A successful simulation course on this topic must focus on creating digestible modules, objective and testable outcomes, and varying difficulty. ${ }^{43}$ Additionally, it should include pitfalls and failures, so that trainees become accustomed to identifying problems. For example, one potential objective outcome is time-to-pedicle screw instrumentation. ${ }^{44}$ An example of a potential pitfall is a registration error, creating inaccurate navigation.

\section{PROPOSED ROBOTIC SURGERY CURRICULUM}

As discussed in the preceding sections, with increasing utilization of spine surgery robots, ${ }^{45,46}$ it is imperative that the field ensures that spine surgery robots are employed safely vis-à-vis training. Given that the learning curve is approximately 15 to 50 cases for all modern systems, ${ }^{11,47-51}$ it seems imprudent to expect that the current standard of a weekend in-service will be adequate to provide surgeons with the tools necessary to be effective users. Consequently, there is a need for a robotic spine surgery curriculum in neurosurgical and orthopaedic surgery residencies. Similar curricula have been created for urology, general surgery, and obstetrics and gynecology programs. ${ }^{29,30}$ To this end, leaders from multiple surgical professional societies created a template that could be used in the construction of robotic surgery skills curricula. ${ }^{28}$ In line with this, several training programs ${ }^{29,30}$ have published their robotic surgery curricula, which include didactic sessions, dry labs, and live surgery participation. The incorporation of such curricula may (1) equip learners to become safe and effective 
users of robotic systems and (2) make residents more attractive to prospective employers and fellowship directors. ${ }^{52}$ Using general surgery curricula as a template, we propose that a robotic surgery curriculum should include the components outlined below.

\section{Phase 1-Didactic}

The first phase should be designed to acquaint trainees with the core features of the robotic system (eg, the touch screen interface, the effector arm), the fundamental principles of navigated surgery (eg, how to register the system, how to verify accuracy), and the indications and limitations of the systems. Given that several systems are currently on the market, each training program's curriculum may have to be tailored to the system available at that institution.

The core features should first be covered with didactic sessions. These sessions should cover the basics of a navigation system (eg, the patient reference device, registering the system, verifying intraoperative accuracy, and troubleshooting a situation in which patient registration is lost). They should additionally cover how robotic systems build upon freehand instrumentation and how image registration is performed by the robot. This includes fundamental aspects of the technology, such as the impact of the robotic arm on haptic feedback, the imaging sequences necessary to register the robot to the patient, and the core principles of screw trajectory planning. Additionally, the applications (ie, pedicle screw instrumentation), advantages (eg, improved screw placement accuracy), and limitations (eg, registration failure) of these systems should be presented honestly and openly to ensure that trainees are afforded an objective appraisal.

\section{Phase 2-Preclinical Training}

After the learner has demonstrated sufficient mastery of the core knowledge of robotic surgery, the next phase is to ensure that they are technically capable of performing the key steps in robotic surgery, whether via surgery or simulation. We foresee this being broken into the following tasks that should be executed in a dry or simulated lab: (1) registration of the robotic system and screw planning, (2) placement of pedicle screws and an interbody using an open approach, (3) placement of pedicle screws and an interbody using a minimally invasive robotic approach, and (4) troubleshooting failure scenarios. Importantly, the failure scenarios should include the surgeon identifying surgical integrity by setting up traps. Ideally, trainees would be able to attend in-depth and properly structured in-services with measurable goals which would afford them sufficient time to acquaint themselves. ${ }^{11,47-51}$ Such sessions could potentially be jointly hosted by industry partners, enabling trainees to gain familiarity with all current systems. Additionally, they should be led by current leaders in the field of spinal robotics to avoid the potential conflicts inherent to courses solely sponsored by industry.

The cost of robotic systems is the greatest obstacle to the sessions proposed for this phase of the curriculum, with most systems costing \$1 million to $\$ 1.5$ million per unit. ${ }^{53}$ Consequently, we argue that this stage may benefit from collaboration with industry partners to create low-cost but high-fidelity simulators, like the Mimic dV-Trainer. Additionally, online simulations are feasible and necessary, as they allow trainees to map out the workflow; this process is akin to advanced cardiovascular life support simulations, where the user gets acquainted with optimization by virtually interacting with a robot, patient, and software in an expedited fashion. Online software can also accurately simulate failure scenarios in which the surgeon must troubleshoot.

Virtual and simulated training is important because it can address an important concern: workflow. Concerns with workflow impairment and increased operating time have been shown to be factors preventing widespread acceptance of intraoperative navigation in spine surgery. ${ }^{15,16}$ Smooth, reproducible workflow is essential to efficient, safe, and effective surgery. Workflow is a team effort, and so ideally, training should include nursing staff, operating room technologists, anesthesia staff, and device representatives, as well as include all aspects of the surgical process, from operating room setup (Figure 2) to the role of anesthesia (ie, in holding ventilation during thoracic pedicle screw placement). Additionally, training all staff helps to unload the burden of responsibility from the surgeon as tasks become delegated. Although training all staff might seem to be overly burdensome, much of this education can be performed virtually via online simulations. Ultimately, effective workflow translates into patient safety, as one observant person in the operating room, if properly trained, can potentially catch a 


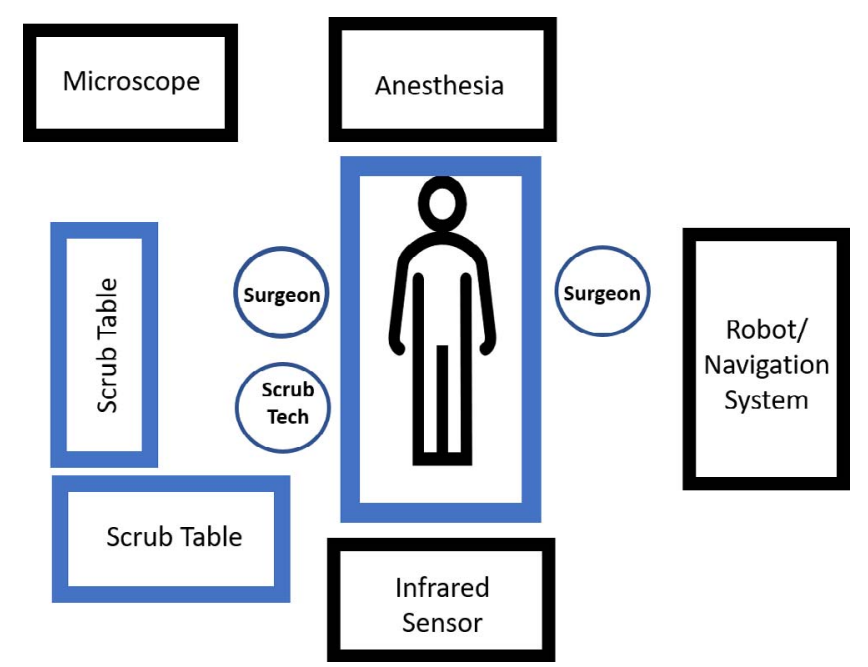

Figure 2. Operating room setup for robotic spine surgery.

critical error. Achieving an effective workflow is therefore contingent upon all team members becoming acquainted with their role in this technology. ${ }^{54}$ Table 1 highlights workflow recommendations for using image guidance and performing robotic spine surgery.

Virtual and simulated training is also an excellent opportunity to address the pitfalls of the technology. An explicit goal during training should be to produce safe surgeons who can recognize technological limitations and troubleshoot problems in real time. In the same way that a resident is not necessarily trained on every procedure in existence but taught the principles of intraoperative troubleshooting, the simulated training should provide the tools needed for a spine surgeon to identify and address red flags and device-related inconsistencies intraoperatively. Some of the most important issues are related to software-based alerts, ensuring accuracy in real time with internal landmarks, and recognizing that preoperative imaging may not match intraoperative anatomy due to progressive disease (eg, tumor or compression fracture), loosening of the spine (eg, after removing ligament or performing an osteotomy), or movement of the patient-mounted tracking frame. Table 2 highlights key pitfalls that surgeons may face and how to troubleshoot. The common problems faced may be divided into 7 categories: registration failure, navigation deviation, tracker or reference frame movement, instrument deflection, issues related to fiducial detection including line of sight, inability to achieve a proper trajectory, and overall system failure. These problems are well documented, and troubleshooting has been previously discussed. ${ }^{54-57}$ Of note, registration may be difficult for patients with high body mass index, severe osteoporosis, or extensive spondylotic changes that obfuscate normal landmarks. In these situations, it is critical that

Table 1. Workflow tips and tricks.

$\begin{array}{ll}\text { Step } & \text { Tips or Trick }\end{array}$

(1) Preoperative planning

(2) Setup

(3) Reference marker placement

(4) Registration

(5) Instrument planning

(6) Robotic surgery or instrument placement
- Ensure all personnel trained

- Setup operating room

- Confirm equipment available

- Review preoperative imaging

- Perform time out

- Drape patient

- Drape robot or system and keep docked out of field

- Strong bony purchase on either end of incision

- In line of view of infrared sensor

- Does not obstruct surgeon

- C-arm or computed tomography moves into position for images then is removed from room - Register patient and instruments to robot or system

- Confirm navigation integrity using anatomical landmarks

- Trajectory for instrument placement planned using navigation system

- Reconfirm navigation integrity

- Bring robot or system into field and dock

- Perform navigated surgery and place instrumentation 
Table 2. Pitfalls and troubleshooting. ${ }^{54-57}$

\begin{tabular}{ll}
\hline Pitfall & \multicolumn{1}{c}{ Solution } \\
\hline Registration failure & - Redo registration process \\
& - Pall for technical support in the case of failure on repeat registration \\
& \\
Navigation deviation & Confirm accuracy of registration frame before instrumentation; reregister if points on \\
& navigation system do not correspond to anatomic landmarks \\
& Verify accuracy of reference frame position in case of questionable navigation accura- \\
& cy $\rightarrow$ if inaccurate, attempt reregistration of reference frame $\rightarrow$ if reregistration un- \\
& successful, convert to freehand instrumentation \\
& Reduce risk of navigation deviation by: \\
& $\circ$ Reducing traction on soft tissues of mobile regions (eg, cervical spine) \\
& $\circ$ Reducing pressure or leaning on patient \\
& $\circ$ Starting instrumentation at level farthest from reference array (site most subject to \\
& navigation deviation secondary to untracked anatomy shift)
\end{tabular}

- Place instrumentation before decompression - the latter may result in loss of bony landmarks used for instrumentation navigation

- Obtain second registration scan (eg, intraoperative computed tomography scan) in long segmental fixation as navigation accuracy decreases with increasing distance from reference frame

- Hold respiratory tidal volume during intraoperative imaging acquisition to increase navigation accuracy (by reducing motion of patient anatomy during scan)

Tracker or reference frame movement or malposition

Instrument skiving

Misalignment secondary to lighting or infrared sensor

Inability to achieve trajectory

System crash
- Avoid contacting the reference frame

- Ensure strong anchorage in bone before initial image registration

- Reduce skin or soft tissue pressure on reference array

- Reattach tracker and repeat image acquisition and tracker registration

- For select systems, employ dual reference array with surveillance marker to monitor for reference frame movement

- Use a burr to drive through cortex and create cannula

- Employ a cortical bone trajectory with entry trajectory orthogonal to surface of bone to avoid skiving

- Consider using a flatter entry trajectory to reduce sheering forces at drill tip

- Select systems has skive meter that may assist in detecting and avoiding intraoperative skive

- Keep reference frame in center of infrared sensor's field of view

- Clean reflective markers on reference frame when sullied by blood or other materials that may alter reflectance

- Avoid overly bright room lighting, which may alter ability of infrared sensor to differentiate reference frame from surrounding objects

- Invert robotic arm or end effector

- Place reference marker facing caudally to avoid obstruction of trajectory

- Convert to open or navigated freehand instrumentation

- Restart robotic system, reregister system or repeat surgical planning

- Convert to open or navigated freehand instrumentation the surgeon be well versed in the anatomy of the sacrum and pelvis to obtain adequate bony purchase for placement of the reference marker. It is of paramount importance to recall that navigation deviation occurs in the setting of bony removal (eg, decompression) and can also occur secondary to instrumentation (eg, interbody cage placement). For these reasons, it is recommended to place instrumentation before bony work when feasible.

$$
\text { Phase 3-Live Surgery }
$$

This third phase is the most straightforward in terms of implementation but is likely the most difficult to achieve, given that not all training 
programs have spine robots. For those that have such systems available, training can proceed in a similar fashion to that used for the training of freehand cases. Residents who have completed the dry lab training sessions will be tasked with sequentially demonstrating the ability to plan screw trajectories using the robotic software, place and register the reference frame, confirm registration accuracy using intraoperative landmarks, place instrumentation in open cases, and place instrumentation in minimally invasive procedures. Programs such as the recently described Surgical Autonomy Program $^{58}$ may help ensure that trainees have completed all crucial steps of this phase. Additionally, trainees who complete a set minimum number of cases may be awarded a certificate certifying their completion of the program.

Live surgery is currently the most common form of training, perhaps skipping Phases 1 and 2 . Although live surgery does facilitate the most rapid learning and simultaneously enables the entire surgical team to train and familiarize themselves with the workflow of the device, it does have limitations that can often be overcome with close attention in Phases 1 and 2. Workflow disruption is commonly cited as one of the main obstacles to the adoption of spine robots, ${ }^{16}$ and a training paradigm that familiarizes all team members with the technology may therefore increase the likelihood of adoption. However, to develop the new workflow necessitates disruption of the previous paradigm, ${ }^{53}$ leading to an initial prolongation of case times. ${ }^{47,59}$ Additionally, such training on the job may potentially risk poorer patient outcomes initially ${ }^{45}$ and may be therefore an inferior strategy if done solely. In a series of 258 patients, Schatlo et $\mathrm{al}^{49}$ noted that there were relative peaks in screw misplacement between cases 10 and 20 and around case 40. The authors argued the initial peak reflects the transition from supervised learner (ie, operating under a surgeon with greater experience with the system) to independent operator. To this end, other authors have recommended that initial cases be performed under supervision of a trained partner. ${ }^{55}$

\section{CONCLUSIONS}

In this paper, we discuss how intraoperative robotics and navigation for spine surgery is safe and beneficial for patients and surgeons but is associated with a learning curve. That learning curve, combined with lack of formal training, is prohibitive for these technologies to become widely embraced and eventually standard of care. Laparoscopic surgery and robotic general surgery have become ubiquitous and the standard of care with the help of low-cost but high-fidelity training modules; thus, so too should the field of spine surgery embrace formal training and education. Training should include the independent creation and implantation of simulated education, with emphasis on objective measurable standards (ie, time to instrumentation), workflow, and pitfalls. At present, the penetration of spine robots within the broader market is too low to mandate the inclusion of training curricula within residency. However, these systems are being rapidly adopted and may create a competitive advantage for surgeons and health systems. ${ }^{60}$ Consequently, as demand rises and the cost of these machines falls, the need to create a standardize training and testing modules becomes all more important to ensure that all robot users have achieved a minimum level of proficiency.

\section{REFERENCES}

1. Walker CT, Kakarla UK, Chang SW, Sonntag VKH. History and advances in spinal neurosurgery. J Neurosurg Spine. 2019;31(6):775-785. doi:10.3171/2019.9.SPINE181362

2. Kochanski RB, Lombardi JM, Laratta JL, Lehman RA, O'Toole JE. Image-guided navigation and robotics in spine surgery. Neurosurgery. 2019;84(6):1179-1189. doi:10.1093/ neuros/nyy630

3. D’Souza M, Gendreau J, Feng A, Kim LH, Ho AL, Veeravagu A. Robotic-assisted spine surgery: history, efficacy, cost, and future trends. Robot Surg. 2019;6:9-23. doi:10.2147/ RSRR.S190720

4. Li HM, Zhang RJ, Shen CL. Accuracy of pedicle screw placement and clinical outcomes of robot-assisted technique versus conventional freehand technique in spine surgery from nine randomized controlled trials: a meta-analysis. Spine (Phila Pa 1976). 2020;45(2):E111-E119. doi:10.1097/BRS. 0000000000003193

5. Fu W, Tong J, Liu G, et al. Robot-assisted technique vs conventional freehand technique in spine surgery: a metaanalysis. Int J Clin Pract. 2021;75(5):e13964. doi:10.1111/ijcp. 13964

6. Li W, Li G, Chen W, Cong L. The safety and accuracy of robot-assisted pedicle screw internal fixation for spine disease: a meta-analysis. Bone Joint Res. 2020;9(10):653-666. doi:10.1302/ 2046-3758.910.BJR-2020-0064.R2

7. Li J, Fang Y, Jin Z, Wang Y, Yu M. The impact of robotassisted spine surgeries on clinical outcomes: a systemic review and meta-analysis. Int $J$ Med Robot. 2020;16(6):1-14. doi:10. $1002 /$ rcs. 2143

8. Ahern DP, Gibbons D, Schroeder GD, Vaccaro AR, Butler JS. Image-guidance, robotics, and the future of spine surgery. Clin Spine Surg. 2020;33(5):179-184. doi:10.1097/BSD. 0000000000000809

9. Overley SC, Cho SK, Mehta AI, Arnold PM. Navigation 
and robotics in spinal surgery: where are we now? Neurosurgery. 2017;80(3S):S86-S99. doi:10.1093/neuros/nyw077

10. Joseph JR, Smith BW, Liu X, Park P. Current applications of robotics in spine surgery: a systematic review of the literature. Neurosurg Focus. 2017;42(5):E2. doi:10.3171/ 2017.2.FOCUS16544

11. Urakov TM, Chang KH, Burks SS, Wang MY. Initial academic experience and learning curve with robotic spine instrumentation. Neurosurg Focus. 2017;42(5):E4. doi:10.3171/ 2017.2.FOCUS175

12. Byun J, Sung T, Park H. Technological innovation strategy: how do technology life cycles change by technological area. Technol Anal Strateg Manag. 2018;30(1):98-112. doi:10. 1080/09537325.2017.1297397

13. Moore GA, Mckenna R. Crossing the Chasm: Marketing and Selling High-Tech Products to Mainstream Customers. New York: HarpersBusiness; 1999.

14. Scott JW. Scott's parabola: the rise and fall of a surgical technique. BMJ. 2001;323:1477. doi:10.1136/bmj.323.7327.1477

15. Virk S, Qureshi S. Navigation in minimally invasive spine surgery. J Spine Surg. 2019;5(Suppl 1):S25-S30. doi:10. $21037 /$ jss.2019.04.23

16. Härtl R, Lam KS, Wang J, Korge A, Kandziora F, Audige L. Worldwide survey on the use of navigation in spine surgery. World Neurosurg. 2013;79(1):162-172. doi:10.1016/j. wneu.2012.03.011

17. Slavin KV. Neuronavigation in neurosurgery: current state of affairs. Expert Rev Med Devices. 2008;5(1):1-3. doi:10. $1586 / 17434440.5 .1 .1$

18. Nano M. A brief history of laparoscopy. G Chir. 2012;33(3):53-57.

19. Antoniou SA, Antoniou GA, Antoniou AI, Granderath FA. Past, present, and future of minimally invasive abdominal surgery. JSLS. 2015;19(3):e2015.00052. doi:10.4293/JSLS.2015. 00052

20. Ballantyne GH. The pitfalls of laparoscopic surgery: challenges for robotics and telerobotic surgery. Surg Laparosc Endosc Percutan Tech. 2002;12(1):1-5. doi:10.1097/00129689200202000-00001

21. Satava RM. The revolution in medical education-the role of simulation. J Grad Med Educ. 2009;1(2):172-175. doi:10. 4300/JGME-D-09-00075.1

22. Kotsis SV, Chung KC. Application of the "see one, do one, teach one" concept in surgical training. Plast Reconstr Surg. 2013;131(5):1194-1201. doi:10.1097/PRS. 0b013e318287a0b3

23. Vassiliou MC, Ghitulescu GA, Feldman LS, et al. The MISTELS program to measure technical skill in laparoscopic surgery: evidence for reliability. Surg Endosc. 2006;20(5):744747. doi:10.1007/s00464-005-3008-y

24. Pugin F, Bucher P, Morel P. History of robotic surgery: from AESOP ${ }^{\circledR}$ and ZEUS ${ }^{\circledR}$ to da Vinci ${ }^{\circledR}$. J Visc Surg. 2011;148(5 Suppl):e3-e8. doi:10.1016/j.jviscsurg.2011.04.007

25. McGuinness LA, Prasad Rai B. Robotics in urology. Ann R Coll Surg Engl. 2018;100(6_sup):38-44. doi:10.1308/ rcsann.supp 1.38

26. Batailler C, Fernandez A, Swan J, et al. MAKO CTbased robotic arm-assisted system is a reliable procedure for total knee arthroplasty: a systematic review. Knee Surg Sports Traumatol Arthrosc. 2020. doi:10.1007/s00167-020-06283-Z

27. Kayani B, Konan S, Huq SS, Tahmassebi J, Haddad FS. Robotic-arm assisted total knee arthroplasty has a learning curve of seven cases for integration into the surgical workflow but no learning curve effect for accuracy of implant positioning. Knee Surg Sports Traumatol Arthrosc. 2019;27(4):1132-1141. doi:10.1007/s00167-018-5138-5

28. Smith R, Patel V, Satava R. Fundamentals of robotic surgery: a course of basic robotic surgery skills based upon a 14society consensus template of outcomes measures and curriculum development. Int J Med Robot. 2014;10(3):379-384. doi:10.1002/rcs.1559

29. Chen R, Rodrigues Armijo P, Krause C, SAGES Robotic Task Force, Siu KC, Oleynikov D. A comprehensive review of robotic surgery curriculum and training for residents, fellows, and postgraduate surgical education. Surg Endosc. 2020;34(1):361-367. doi:10.1007/s00464-019-06775-1

30. Moit H, Dwyer A, De Sutter M, Heinzel S, Crawford D. A standardized robotic training curriculum in a general surgery program. JSLS. 2019;23(4):e2019.00045. doi:10.4293/JSLS. 2019.00045

31. Duffy TP. The Flexner report- 100 years later. Yale $J$ Biol Med. 2011;84(3):269-276.

32. Cohen ER, Feinglass J, Barsuk JH, et al. Cost savings from reduced catheter-related bloodstream infection after simulation-based education for residents in a medical intensive care unit. Simul Healthc. 2010;5(2):98-102. doi:10.1097/SIH 0b013e3181bc8304

33. Rodgers DL, Securro S, Pauley RD. The effect of highfidelity simulation on educational outcomes in an advanced cardiovascular life support course. Simul Healthc. 2009;4(4):200-206. doi:10.1097/SIH.0b013e3181b1b877

34. Ziv A, Wolpe PR, Small SD, Glick S. Simulation-based medical education: an ethical imperative. Acad Med. 2003;78(8):783-788. doi:10.1097/00001888-200308000-00006

35. Graber MA, Wyatt C, Kasparek L, Xu Y. Does simulator training for medical students change patient opinions and attitudes toward medical student procedures in the emergency department? Acad Emerg Med. 2005;12(7):635-639. doi:10.1197/j.aem.2005.01.009

36. Badash I, Burtt K, Solorzano CA, Carey JN. Innovations in surgery simulation: a review of past, current and future techniques. Ann Transl Med. 2016;4(23):453. doi:10.21037/atm. 2016.12.24

37. Schaefer JJ, Vanderbilt AA, Cason CL, et al. Literature review: instructional design and pedagogy science in healthcare simulation. Simul Healthc. 2011;6 Suppl:S30-S41. doi:10.1097/ SIH.0b013e31822237b4

38. Siddiqui NY, Galloway ML, Geller EJ, et al. Validity and reliability of the robotic objective structured assessment of technical skills. Obstet Gynecol. 2014;123(6):1193-1199. doi:10. 1097/AOG.0000000000000288

39. Perrenot C, Perez M, Tran N, et al. The virtual reality simulator $\mathrm{dV}$-Trainer ${ }^{\circledR}$ is a valid assessment tool for robotic surgical skills. Surg Endosc. 2012;26(9):2587-2593. doi:10.1007/ s00464-012-2237-0

40. Tam V, Borrebach J, Dunn SA, Bellon J, Zeh HJ, Hogg ME. Proficiency-based training and credentialing can improve patient outcomes and decrease cost to a hospital system. Am J Surg. 2019;217(4):591-596. doi:S0002-9610(18)30543-9

41. Kirkman MA, Ahmed M, Albert AF, Wilson MH, Nandi D, Sevdalis N. The use of simulation in neurosurgical education and training. A systematic review. J Neurosurg. 2014;121(2):228-246. doi:10.3171/2014.5.JNS131766

42. Bohm PE, Arnold PM. Simulation and resident 
education in spinal neurosurgery. Surg Neurol Int. 2015;6:33. doi:10.4103/2152-7806.152146

43. McGaghie WC, Issenberg SB, Petrusa ER, Scalese RJ. Effect of practice on standardised learning outcomes in simulation-based medical education. Med Educ. 2006;40(8):792-797. doi:10.1111/j.1365-2929.2006.02528.x

44. Park HJ, Wang C, Choi KH, Kim HN. Use of a life-size three-dimensional-printed spine model for pedicle screw instrumentation training. J Orthop Surg Res. 2018;13(1):86. doi:10. 1186/s13018-018-0788-z

45. Fiani B, Quadri SA, Farooqui M, et al. Impact of robotassisted spine surgery on health care quality and neurosurgical economics: a systemic review. Neurosurg Rev. 2020;43(1):17-25. doi:10.1007/s10143-018-0971-z

46. Transparency Market Research. Spine surgery robots market (spinal fusion, minimally invasive procedures, scoliosis, osteoporotic compression fractures) - global industry analysis, size, share, growth, trends, and forecast, 2018-2026. Global Information, Inc Web site. https://www.giiresearch.com/report/ tsm769602-spine-surgery-robots-market-spinal-fusion.html. Updated 2018. Accessed February 15, 2021.

47. Jiang B, Pennington Z, Azad T, et al. Robot-assisted versus freehand instrumentation in short-segment lumbar fusion: experience with real-time image-guided spinal robot. World Neurosurg. 2020;136:e635-e645. doi:10.1016/j.wneu. 2020.01.119

48. Passias PG, Brown AE, Alas H, et al. A cost benefit analysis of increasing surgical technology in lumbar spine fusion. Spine J. 2021;21(2):193-201. doi:10.1016/j.spinee.2020. 10.012

49. Schatlo B, Martinez R, Alaid A, et al. Unskilled unawareness and the learning curve in robotic spine surgery. Acta Neurochir (Wien). 2015;157(10):1819-1823; discussion 1823. doi:10.1007/s00701-015-2535-0

50. Khan A, Meyers JE, Siasios I, Pollina J. Next-generation robotic spine surgery: First report on feasibility, safety, and learning curve. Oper Neurosurg (Hagerstown). 2019;17(1):6169. doi:10.1093/ons/opy 280

51. Hu X, Lieberman IH. What is the learning curve for robotic-assisted pedicle screw placement in spine surgery? Clin Orthop Relat Res. 2014;472(6):1839-1844. doi:10.1007/s11999013-3291-1

52. Kapadia S, Shellito A, Tom CM, et al. Should robotic surgery training be prioritized in general surgery residency? A survey of fellowship program director perspectives. J Surg Educ. 2020;77(6):e245-e250. doi:S1931-7204(20)30259-2

53. Hussain I, Cosar M, Kirnaz S, et al. Evolving navigation, robotics, and augmented reality in minimally invasive spine surgery. Global Spine J. 2020;10(2 Suppl):22S-33S. doi:10.1177/ 2192568220907896

54. Tian W, Liu YJ, Liu B, et al. Guideline for thoracolumbar pedicle screw placement assisted by orthopaedic surgical robot. Orthop Surg. 2019;11(2):153-159. doi:10.1111/ os. 12453

55. Garg B, Mehta N, Malhotra R. Robotic spine surgery: ushering in a new era. J Clin Orthop Trauma. 2020;11(5):753760. doi:10.1016/j.jcot.2020.04.034

56. Buza JA, Good CR, Lehman RA, et al. Robotic-assisted cortical bone trajectory (CBT) screws using the mazor X stealth edition (MXSE) system: workflow and technical tips for safe and efficient use. J Robot Surg. 2021;15(1):13-23. doi:10.1007/ s11701-020-01147-7

57. Crawford N, Johnson N, Theodore N. Ensuring navigation integrity using robotics in spine surgery. $J$ Robot Surg. 2020;14(1):177-183. doi:10.1007/s11701-019-00963-w

58. Haglund MM, Cutler AB, Suarez A, Dharmapurikar R, Lad SP, McDaniel KE. The surgical autonomy program: a pilot study of social learning theory applied to competency-based neurosurgical education. Neurosurgery. 2021;88(4):E345-E350. doi:10.1093/neuros/nyaa556

59. Lieberman IH, Kisinde S, Hesselbacher S. Roboticassisted pedicle screw placement during spine surgery. JBJS Essent Surg Tech. 2020;10(2):e0020. doi:10.2106/JBJS.ST.19. 00020

60. Chen R, Rodrigues Armijo P, Krause C, SAGES Robotic Task Force, Siu KC, Oleynikov D. A comprehensive review of robotic surgery curriculum and training for residents, fellows, and postgraduate surgical education. Surg Endosc. 2020;34(1):361-367. doi:10.1007/s00464-019-06775-1

Disclosures and COI: Nicholas Theodore has intellectual property and receives royalties from Globus Medical and Depuy Synthes. He is a paid consultant to Globus Medical and own Globus Medical stock. The remaining authors have nothing to disclose.

IRB Approval: The contents of this manuscript do not meet National Institutes of Health definitions for human subject research. The Institutional Review Board (IRB) at Johns Hopkins University School of Medicine deemed that IRB approval was not required for this work.

Corresponding Author: Nicholas Theodore, MD, Johns Hopkins University School of Medicine, 600 N. Wolfe St, Meyer 7-113, Baltimore, MD 21287. Phone: (602) 549-7594; Fax: (410) 502-3399; Email: theodore@jhmi.edu.

Published 26 October 2021

This manuscript is generously published free of charge by ISASS, the International Society for the Advancement of Spine Surgery. Copyright (C) 2021 ISASS. To see more or order reprints or permissions, see http://ijssurgery.com. 